\title{
Doença hepática gordurosa não alcoólica
}

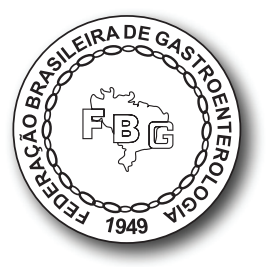

Claudia Pinto Marques Souza de Oliveira

José Tadeu Stefano

\section{INTRODUÇÃO}

A doença hepática gordurosa não alcoólica (DHGNA) é uma das formas mais comuns de doença hepática, relacionada primordialmente ao aumento progressivo da obesidade no mundo. Inicialmente, foi considerada uma hepatopatia de curso benigno, contudo, atualmente, sabe-se que é uma doença complexa que envolve fatores ambientais e predisposição genética ${ }^{1}$.

A DHGNA abrange um espectro de alterações hepáticas que variam desde um simples depósito de gordura no interior do hepatócito, sem inflamação ou fibrose (esteatose simples), até casos de esteato-hepatite não alcoólica (EHNA), cirrose e carcinoma hepatocelular, em pacientes sem história de etilismo ${ }^{2}$. A EHNA propriamente dita foi descrita inicialmente na década de 1980 por Ludwig et al. e é definida como uma entidade clinicopatológica caracterizada por alterações histológicas que se assemelham àquelas encontradas na doença hepática alcoólica (DHA), como: esteatose macro e microvesicular, infiltrado inflamatório lobular misto e balonização hepatocelular em área da veia centrolobular (zona III), podendo apresentar fibrose pericelular, corpúsculos de Mallory e cirrose. Esses aspectos morfológicos são indiferenciáveis da DHA, no entanto, ocorrem em indivíduos cujo consumo diário de álcool é inferior a $20 \mathrm{~g}$ para mulheres e $40 \mathrm{~g}$ para homens $\mathrm{s}^{3,4}$.

Em decorrência das crescentes taxas de obesidade, a DHGNA tem se tornado cada vez mais frequente em todas as populações, principalmente no mundo ocidental e tem sido definida pelos especialistas como uma doença do mundo moderno ${ }^{5}$. A DHGNA e a EHNA são altamente prevalentes nos Estados Unidos e em todo o mundo. No entanto, essa prevalência difere significativamente 
de acordo com o método diagnóstico utilizado e a população estudada. À medida que as taxas de obesidade, diabetes e síndrome metabólica (SM) continuam a aumentar, a DHGNA e a EHNA podem afetar significativamente os cuidados de saúde e o desenvolvimento de complicações a longo prazo, como cirrose e carcinoma hepatocelular nos próximos anos ${ }^{6}$.

Existem poucos estudos epidemiológicos sobre prevalência da DHGNA na América do Sul. No Brasil, Karnikowski et al. ${ }^{7}$ relataram que a prevalência da DHGNA é de 35,2\% entre a população de meia-idade. Outro estudo realizado pela Sociedade Brasileira de Hepatologia avaliou as características clinicoepidemiológicas da DHGNA em 16 centros médicos distribuídos pelas cinco regiões do Brasil. Os dados desse estudo mostraram que a maioria dos indivíduos era do sexo masculino (53,3\%), 85\% eram assintomáticos e a maioria (58\%) já apresentava EHNA e, desses, 27\% apresentavam algum grau de fibrose, incluindo $15,4 \%$ de casos de cirrose ${ }^{8}$.

\section{FISIOPATOLOGIA}

A patogênese da DHGNA ainda não está bem esclarecida, no entanto, os fatores de risco são bem conhecidos e estão relacionados principalmente à SM [obesidade, diabetes mellitus tipo 2, resistência à insulina (RI), dislipidemia $\mathrm{e}$ hipertensão]. Embora se conheçam os fatores predisponentes e se saiba que a esteatose pode evoluir para cronicidade, a verdadeira relação causal entre esteatose/EHNA, fibrogênese e doença crônica do fígado, assim como sua patogênese, ainda não está totalmente esclarecida. Dentre as hipóteses consideradas para explicar a fisiopatogênese da DHGNA e sua evolução para EHNA, destaca-se a teoria dos múltiplos hits, que aponta a RI como condição inicial para o acúmulo de ácidos graxos no hepatócito (esteatose) ${ }^{9,10}$ e os estímulos subsequentes, como estresse oxidativo, ativação de citocinas inflamatórias, estresse do retículo endotelial, entre outros (múltiplos hits), para o desenvolvimento de inflamação e fibrose ${ }^{11,12}$. Outros fatores endógenos como a microbiota intestinal também podem contribuir para o desenvolvimento da DHGNA. $\mathrm{O}$ aumento da permeabilidade intestinal e o supercrescimento bacteriano do intestino delgado (SCBID), que é uma condição na qual níveis elevados do número de bactérias do intestino delgado levam à distensão abdominal e outros sintomas comuns relacionados com síndrome do intestino irritável (SII), são frequentes em pacientes obesos e induzem a lesão hepática por aumentar a produção de lipopolissacarídeos (LPS) derivados das bactérias Gram-negativas do intestino e a produção de TNF-alfa. Também sugerem que a microbiota intestinal aumenta a exposição do fígado a endotoxinas desempenhando um papel importante na progressão da esteatose para EHNA ${ }^{13-15}$. 


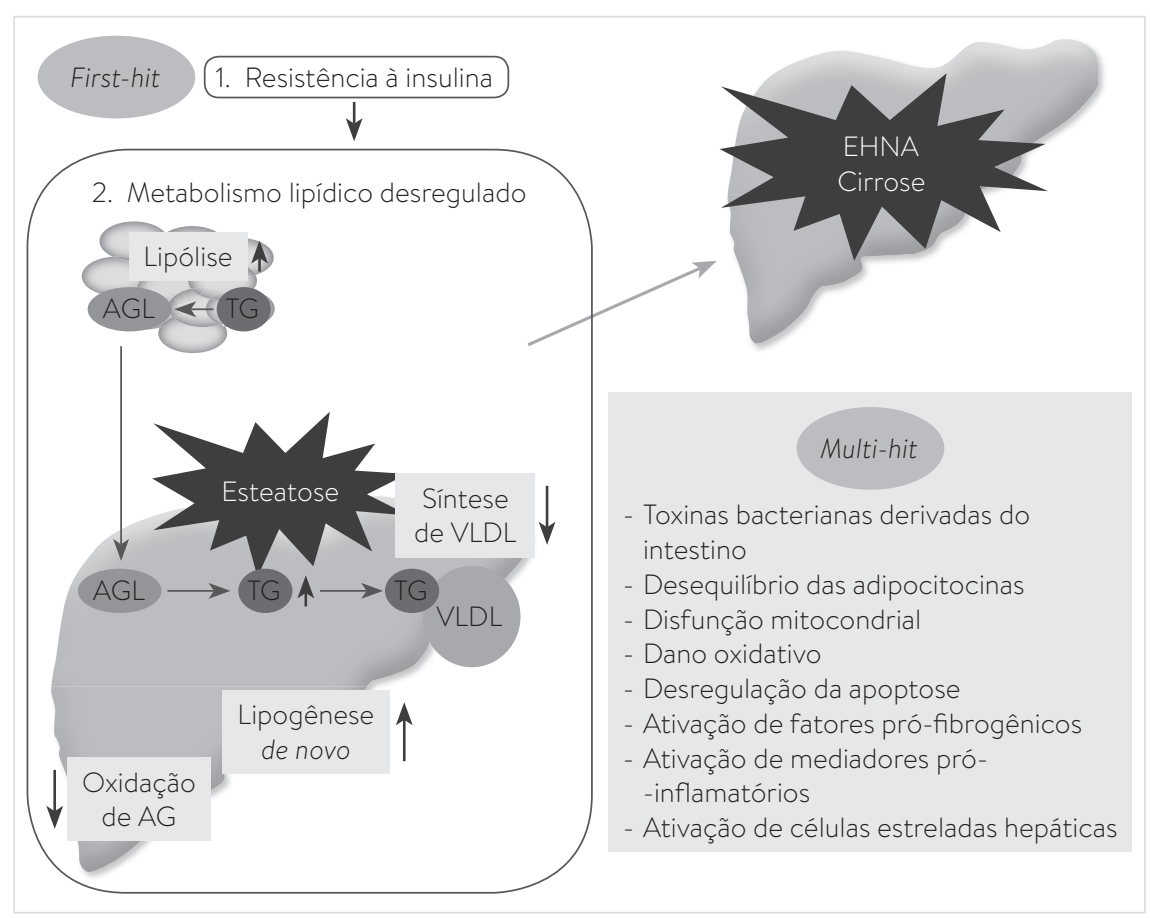

Figura 1 Fatores que influenciam a severidade da esteatose, progressão para esteato-hepatite, fibrose e cirrose ${ }^{16}$.

AGL: ácidos graxos livres; TG: triglicérides; VLDL: lipoproteína de densidade muito baixa.

\section{DIAGNÓSTICO}

A DHGNA é usualmente assintomática. Em geral, os pacientes não apresentam queixas nos estágios iniciais da doença e somente começam a ter sintomas, como fadiga, desconforto no quadrante superior direito do abdome, perda de peso e fraqueza, quando a doença já está mais avançada ou há a presença de cirrose. A doença hepática é muitas vezes descoberta acidentalmente durante exames laboratoriais de rotina, que revelam concentrações aumentadas de alanina aminotransferase (ALT), ou durante investigação de pacientes com obesidade grave, DM e/ou hipertensão arterial, comorbidades que representam fatores de risco para desenvolvimento da DHGNA. A anormalidade mais comum no exame físico desses pacientes é o sobrepeso (IMC $>25 \mathrm{~kg} / \mathrm{m}^{2}$ ) (56 a 79\%), seguido de hipertensão arterial e adiposidade visceral. No entanto, tem-se observado que indivíduos com DHGNA e com o IMC dentro da normalidade também podem apresentar adiposidade visceral. Hepatomegalia é 
descrita como sinal comum nos pacientes com DHGNA e esse número aumenta quando avaliado por ultrassom. Sinais e sintomas de insuficiência hepática, como spider angiomata, ginecomastia e eritema palmar, ocorrem menos frequentemente que em outras hepatopatias crônicas, embora a esplenomegalia possa ocorrer em até $25 \%$ dos pacientes na época do diagnóstico.

O diagnóstico da DHGNA é baseado na exclusão de outras causas de doença hepática. Uma vez descartadas condições como as hepatites virais, álcool, fatores genéticos e ambientais, o diagnóstico de DHGNA primária pode ser considerado. Elevações leves a moderadas de aminotransferases, usualmente menores que quatro vezes o limite superior da normalidade, são a anormalidade laboratorial mais comum e são frequentemente encontradas em pacientes com DHGNA. A DHGNA é a causa mais comum para a inexplicada e persistente elevação das concentrações de ALT, embora alguns pacientes possam apresentar enzimas hepáticas normais, mesmo que tenham evoluído para EHNA. Já a aspartato aminotransferase (AST) apresenta valores inferiores aos da ALT, diferindo da DHA. Em metade dos pacientes com DHGNA coexiste elevação da GGT, algumas vezes sendo a única enzima hepática elevada. Hiperbilirrubinemia, hipoalbuminemia e tempo de protrombina prolongado são infrequentes e, geralmente, são observados quando a falência hepática já está bem estabelecida. Elevações no perfil lipídico sérico e nas concentrações de glicose são comuns em pacientes com DHGNA e têm sido relatadas em cerca de 25 a $75 \%$ dos casos.

Conforme já mencionado anteriormente, a DHGNA, na maioria das vezes, é suspeitada ou descoberta por acaso em avaliações de rotina, normalmente por meio de ultrassonografia (USG) simples de abdome ou exames laboratoriais abordando enzimas hepáticas. O exame inicial é USG abdominal, por ser de baixo custo e amplamente disponível. Deve-se salientar, contudo, que ele tem sensibilidade limitada para detectar esteatose quando em menos de $20 \%$ ou em obesos com IMC $>40 \mathrm{~kg} / \mathrm{m}^{2}$. A espectroscopia por ressonância magnética, exame de imagem ideal para a detecção de DHGNA, é usada apenas em centros específicos para propósitos de estudo. Outra forma de se abordar de maneira não invasiva a DHGNA são os escores clínicos para esteatose, e três deles têm ganhado relevância por já terem sido validados externamente, apesar de servirem apenas para estabelecer a presença, não a gravidade: o índice de gordura hepática (FLI, do inglês fatty liver index); o SteatoTest ${ }^{\circledR}$; e o escore de gordura hepática para DHGNA (em inglês, NAFLD liver fat score) ${ }^{17}$.

Para diagnóstico de fibrose, grande determinante do prognóstico na DHG$\mathrm{NA}$, foram também desenvolvidos escores, sendo os mais bem estabelecidos, já validados externamente, o escore de fibrose na DHGNA (NAFLD fibrosis score - NFS) e o calculador de fibrose 4 (FIB-4), além do FibroTest ${ }^{\circledR}$, já usado 
anteriormente em hepatite crônica B e C para estabelecer fibrose avançada. Estes testes são mais acurados para distinguir fibrose avançada (F3 ou maior) de não avançada ${ }^{18}$.

Outra ferramenta de avaliação não invasiva é a elastografia transitória (FibroScan $\left.{ }^{\circledR}\right)$, também já de grande uso nas hepatites virais crônicas. Assim como outros testes, o desempenho é melhor para diferenciar fibrose avançada de não avançada. O parâmetro de atenuação controlada (controlled attenuation parameter - CAP) pode ser medido junto à elastografia e mostrou-se capaz de detectar esteatose com boa acurácia, mas é menos confiável para a determinação do grau de acometimento ${ }^{18}$.

Ainda não existe consenso quanto ao uso de todos esses marcadores para a avaliação inicial e continuada da DHGNA, especialmente em se tratando de evitar biópsia hepática. Muitos autores sugerem a combinação de vários desses métodos. Para o diagnóstico de EHNA, ainda é necessária a biópsia. A decisão de se propor a biópsia deve ser discutida e individualizada com cada paciente. Vários achados clínicos associados à EHNA e/ou à fibrose avançada em pacientes com DHGNA podem auxiliar na indicação de biópsia hepática, dentre eles: idade acima de 45 anos, obesidade ou diabetes e relação AST/ALT $>1$. As indicações da biópsia hepática são: alteração de enzimas hepáticas ou dos escores de fibrose no contexto de esteatose (normalmente vista em USG), ou ainda para diferenciar o tipo de acometimento hepático predominante em situações de doenças concomitantes. O estudo anatomopatológico ainda é o mecanismo ideal para se estadiar a doença. Os achados típicos da EHNA são: a) esteatose, normalmente macrogoticular e graduada em três níveis; b) balonização de hepatócitos; c) infiltrado inflamatório lobular misto; e d) fibrose, inicialmente perissinusoidal, predominando em zona 3 (zona centrolobular), podendo ainda haver fibrose periportal. A fibrose, contudo, não é necessária para se firmar o diagnóstico. Além desses, pode haver inflamação periportal, vacuolização glicogênica, megamitocôndrias e corpúsculos de Mallory-Denk, em número menor que na doença alcoólica, a qual, por sua vez, é em muitos aspectos idêntica, apesar de ter inflamação lobular mais exuberante e outras características próprias ${ }^{18}$.

Dois sistemas de graduação (escores) são mais utilizados na avaliação da EHNA: o NAS (NASH activity score, escore de atividade da EHNA) e o SAF (steatosis activity and fibrosis, fibrose e atividade na esteatose). Ambos se baseiam no grau de esteatose, de balonização e de infiltrado inflamatório, além da fibrose, sendo esta pontuada à parte no NAS. Um valor de 5 para o NAS era tradicionalmente usado para se considerar EHNA, entretanto, orientações mais recentes sugerem que o diagnóstico deve se basear na avaliação patológica geral, devendo-se usar os escores para a graduação da doença apenas. $\mathrm{O}$ 
NAS, contudo, não se mostrou adequado para prognóstico; o SAF ainda não tem relevância clínica ou prognóstica determinada ${ }^{19}$.

\section{TRATAMENTO}

Os objetivos do tratamento da DHGNA seriam, a longo prazo, redução da progressão da lesão hepática, principalmente para cirrose, redução da incidência de carcinoma hepatocelular e redução de mortalidade.

Mudanças dos hábitos de vida, como dieta pobre em lipídios e de baixo teor calórico e o incentivo à prática de atividade física, devem ser instituídos para todos os pacientes. O principal objetivo consiste em perda ponderal e melhora da composição corporal, com redução da massa gorda e aumento proporcional de massa magra. A perda de peso é a medida mais eficaz de controle da doença, contudo, para que seja eficaz na melhora da histologia hepática, esta deve ser de pelo menos 7 a 10\% do peso inicial, com redução dos graus de esteatose e inflamação, assim como redução dos níveis séricos das aminotransferases.

Tanto os exercícios aeróbicos como os treinos de resistência parecem ser efetivos no controle do depósito de gordura no fígado, porém a prática regular e contínua é necessária para que se consiga atingir esse objetivo. $\mathrm{O}$ papel da dieta e do exercício físico não se limita à redução do depósito de gordura nos hepatócitos, mas essa abordagem em conjunto também aumenta a sensibilidade à insulina. Para pacientes com obesidade grave (IMC $>35 \mathrm{~kg} / \mathrm{m}^{2}$ ) e que têm EHNA, a cirurgia bariátrica é uma opção a ser considerada, visto que para a maioria desses pacientes dieta e atividade física não são eficazes. Uma vez que uma perda de peso rápida pode piorar a histologia hepática, as cirurgias bariátricas que utilizam bypass jejunoileal devem ser evitadas e gastroplastias ou bandas ajustáveis devem ser preferidas.

A terapia farmacológica pode ser utilizada em pacientes com EHNA e que têm risco para evoluir para formas mais graves. Ainda é uma questão em aberto quais pacientes com EHNA são elegíveis para terapia farmacológica específica. Pacientes com fibrose (F2) apresentam alto risco de progressão e, portanto, necessitam de terapias dirigidas ao fígado. Indiscutivelmente, pacientes em fases anteriores, mas com fatores de risco acumulado para fibrose hepática (diabetes, idade acima de 50, hipertensão arterial, ou RI grave) também poderiam ser candidatos a esses tratamentos. Por último, é importante que uma abordagem integrada que combine medidas farmacológicas e não farmacológicas seja cuidadosamente planejada para avaliações futuras ${ }^{20}$.

Dentre as drogas que têm propriedade antioxidante, a vitamina E está entre as mais estudadas e utilizadas. Seu uso ganhou impulso após o estudo PIVENS publicado no New England Journal of Medicine (NEJM) em 2010, em que 
Sanyal et al. mostraram melhora do NAS com o uso de vitamina E. A dose preconizada deve ser de $800 \mathrm{UI} /$ dia, porém seu uso deve ser restrito aos pacientes não cirróticos e não diabéticos, pois ainda não existe evidência suficiente para ser indicada nos portadores de DM nem nos pacientes com cirrose ${ }^{21}$. Ainda não se conhece o tempo ideal de uso, porém sabe-se que o uso prolongado de vitamina $\mathrm{E}$ deve ser evitado, pois foram observados aumento da incidência de câncer de próstata em homens acima de 50 anos e aumento da incidência de acidente vascular cerebral (AVC) hemorrágico, além de um aumento da mortalidade geral. Nos pacientes que antes do tratamento têm enzimas hepáticas elevadas, a não redução dos seus níveis após 6 meses de uso pode ser utilizada como critério de suspensão.

Agentes sensibilizadores de insulina são as tiazolinedionas, sendo a pioglitazona a mais estudada na EHNA. A pioglitazona é um agonista do receptor $\gamma$ de peroxissomo proliferador-ativado (PPAR- $\gamma$ ) encontrado no tecido adiposo, músculo esquelético e fígado. Quando ativados, esses receptores modulam a transcrição de genes que alteram a sensibilização à insulina. Dessa forma, age reduzindo a resistência à insulina na periferia, além de reduzir a produção de glicose pelo fígado. O estudo PIVENS já citado comparou o uso de pioglitazona na dose de $30 \mathrm{mg} /$ dia por 2 anos em pacientes não cirróticos e não diabéticos com o placebo e evidenciou melhora histológica, tanto na balonização como na inflamação e esteatose, mas sem melhora no grau de fibrose hepática. Efeitos colaterais relatados com o uso da droga a longo prazo são ganho ponderal, aumento na incidência de fraturas e insuficiência cardíaca congestiva. Assim como a vitamina $\mathrm{E}$ usada isoladamente, a pioglitazona pode ser utilizada como alternativa ou ambas podem ser utilizadas em associação nos pacientes com EHNA, mas efeitos a longo prazo ainda são desconhecidos e o tempo de tratamento seguro permanece obscuro.

A metformina atua inibindo a gliconeogênese hepática e reduzindo a absorção de glicose, o que leva a maior captação da glicose pelas células musculares. Vários estudos utilizando a metformina em pacientes com EHNA evidenciaram perda de peso, diminuição da RI, melhora dos índices bioquímicos de lesão hepatocelular, contudo metanálise publicada no Hepatology em 2010 mostrou que a metformina não foi efetiva na melhora histológica ou no nível das transaminases. Contudo, pode ser utilizada como adjuvante em pacientes com diabetes ou com glicemia de jejum alterada/intolerância à glicose, em associação com vitamina E e/ou glitazonas. Alguns trabalhos e metanálise recente têm demonstrado que a metformina reduz a incidência de $\mathrm{CHC}$.

O uso das estatinas, ômega 3 e outras substâncias hipolipemiantes é permitido nos pacientes com DHGNA e deve ser estimulado nos pacientes portadores de dislipidemia. Contudo, até a presente data, entretanto, não há estu- 
dos que mostrem benefício direto desses fármacos na melhora histológica da EHNA.

Estudos de fase 3 com drogas promissoras (Tabela 1) estão em andamento para determinar seu uso a longo prazo. O ácido obeticólico, derivado sintético do ácido quenodenoxicólico, agonista natural do receptor nuclear farnesoide $\mathrm{X}$ (FXR), o elafibranor, agonista PPAR e o cenicriviroc (antagonista de quimiocinas) demonstraram em estudos de fase II melhora histológica, com redução do NAS sem piora do grau de fibrose. Entretanto, estudos de acompanhamento a longo prazo e melhor conhecimento sobre o perfil de segurança das drogas são necessários para que se passe a utilizar essa medicação na prática clínica diária ${ }^{22}$.

Análogos da incretina, como a liraglutida, parecem ser terapias promissoras. São agonistas do receptor GLP-1 (glucagon-like protein 1) que, quando ativados, levam ao aumento da secreção de insulina, redução da secreção inapropriada do glucagon, redução da velocidade de esvaziamento gástrico e consequente saciedade precoce. O estudo LEAN publicado no Lancet em 2016 mostrou melhora bioquímica e histológica em pacientes que utilizaram liraglutida em um acompanhamento de 48 semanas em comparação ao placebo. Novos estudos são necessários para confirmação desse benefício ${ }^{23}$.

Finalmente, há necessidade imediata de desenvolvimento de terapia medicamentosa para EHNA e, nos Estados Unidos e na Europa, alguns trials clínicos estão em andamento nesse sentido. Resumidamente, observam-se na Tabela 1 os principais trials.

\begin{tabular}{|c|c|c|c|c|c|}
\hline Agente & $\begin{array}{l}\text { Descrição } \\
\text { do estudo }\end{array}$ & Duração & $\begin{array}{l}\text { Número de } \\
\text { pacientes }\end{array}$ & População & $\begin{array}{l}\text { Número do } \\
\text { Clinical trials }\end{array}$ \\
\hline EPA-E & $\begin{array}{l}600 \text { mg vs. } \\
900 \text { mg } \\
\text { placebo oral }\end{array}$ & $\begin{array}{l}52 \\
\text { semanas }\end{array}$ & 243 & Não cirróticos & NCT01154985 \\
\hline $\begin{array}{l}\text { Ácido } \\
\text { obeticólico }\end{array}$ & $\begin{array}{l}25 \text { mg vs. } \\
\text { placebo oral }\end{array}$ & $\begin{array}{l}72 \\
\text { semanas }\end{array}$ & 280 & Não cirróticos & NCT01265498 \\
\hline $\begin{array}{l}\text { Simtuzumab } \\
\text { (GS6624) }\end{array}$ & $\begin{array}{l}75 \text { mg vs. } 120 \\
\text { mg vs. placebo } \\
\text { intravenoso }\end{array}$ & $\begin{array}{l}96 \\
\text { semanas }\end{array}$ & 225 & Cirrose & NCT016722866 \\
\hline $\begin{array}{l}\text { Simtuzumab } \\
\text { (GS6624) }\end{array}$ & $\begin{array}{l}200 \text { mg vs. } \\
700 \text { mg vs. } \\
\text { placebo }\end{array}$ & $\begin{array}{l}96 \\
\text { semanas }\end{array}$ & 225 & $\begin{array}{l}\text { Fibrose } \\
\text { avançada sem } \\
\text { cirrose }\end{array}$ & NCT01672879 \\
\hline
\end{tabular}




\begin{tabular}{|c|c|c|c|c|c|}
\hline Agente & $\begin{array}{l}\text { Descrição } \\
\text { do estudo }\end{array}$ & Duração & $\begin{array}{l}\text { Número de } \\
\text { pacientes }\end{array}$ & População & $\begin{array}{l}\text { Número do } \\
\text { Clinical trials }\end{array}$ \\
\hline Elafibranor & $\begin{array}{l}80 \text { mg vs. } 120 \\
\text { mg vs. placebo }\end{array}$ & $\begin{array}{l}52 \\
\text { semanas }\end{array}$ & 270 & Não cirróticos & NCT01694849 \\
\hline Liraglutide & $\begin{array}{l}1,8 \text { mg vs } \bigcirc D \\
\text { vs. placebo }\end{array}$ & $\begin{array}{l}48 \\
\text { semanas }\end{array}$ & 50 & $\begin{array}{l}\text { Diabéticos e } \\
\text { não diabéticos }\end{array}$ & NCT012377119 \\
\hline Losartan & $\begin{array}{l}50 \text { mg vs. } \\
\text { plabebo }\end{array}$ & 2 anos & 214 & $\begin{array}{l}\text { EHNA com } \\
\text { fibrose }\end{array}$ & NCT01051219 \\
\hline Cenicriviroc & $\begin{array}{l}150 \text { mg vs. } \\
\text { placebo }\end{array}$ & 2 anos & 252 & $\begin{array}{l}\text { EHNA com } \\
\text { fibrose }\end{array}$ & NCT002217475 \\
\hline Arachmol & $\begin{array}{l}400 \text { mg vs. } \\
600 \text { mg vs. } \\
\text { placebo }\end{array}$ & 1 ano & 240 & $\begin{array}{l}\text { EHNA com } \\
\text { pré-diabetes } \\
\text { ou diabetes } \\
\text { e obesidade } \\
\text { visceral }\end{array}$ & NCT002279524 \\
\hline
\end{tabular}

Fonte: www.clinicaltrials.gov.

\section{REFERÊNCIAS BIBLIOGRÁFICAS}

1. Day CP, Saksena S. Non-alcoholic steatohepatitis: definitions and pathogenesis. J Gastroenterol Hepatol. 2002;17(Suppl3):S377-384.

2. Farrell GC, Larter CZ. Nonalcoholic fatty liver disease: from steatosis to cirrhosis. Hepatology. 2006;43:S99-S112.

3. Brunt EM, Janney CG, Di Bisceglie AM, Neuschwander-Tetri BA, Bacon BR. Nonalcoholic steatohepatitis: a proposal for grading and staging the histological lesions. Am J Gastroenterol. 1999;94:2467-74.

4. Youssef WI, McCullough AJ. Steatohepatitis in obese individuals. Best Pract Res Clin Gastroenterol. 2002;16:733-47.

5. Angelico F, Del Ben M, Conti R, Francioso S, Feole K, Fiorello S, et al. Insulin resistance, the metabolic syndrome, and nonalcoholic fatty liver disease. J Clin Endocrinol Metab. 2005;90:1578-82.

6. Sayiner M, Koenig A, Henry L, Younossi ZM. Epidemiology of nonalcoholic fatty liver disease and nonalcoholic steatohepatitis in the United States and the rest of the world. Clin Liver Dis. 2016;20:205-14.

7. Karnikowski M, Córdova C, Oliveira RJ, Karnikowski MG, Nóbrega OeT. Non-alcoholic fatty liver disease and metabolic syndrome in Brazilian middle-aged and older adults. Sao Paulo Med J. 2007;125:333-7.

8. Cotrim HP, Parise ER, Oliveira CP, Leite N, Martinelli A, Galizzi J, et al. Nonalcoholic fatty liver disease in Brazil. Clinical and histological profile. Ann Hepatol. 2011;10:33-7.

9. Scheen AJ, Luyckx FH. Nonalcoholic steatohepatitis and insulin resistance: interface between gastroenterologists and endocrinologists. Acta Clin Belg. 2003;58:81-91.

10. Chitturi S, Abeygunasekera S, Farrell GC, Holmes-Walker J, Hui JM, Fung C, et al. NASH and insulin resistance: Insulin hypersecretion and specific association with the insulin resistance syndrome. Hepatology. 2002;35:373-9. 
11. McCullough AJ. Pathophysiology of nonalcoholic steatohepatitis. J Clin Gastroenterol. 2006;40(Suppl1):S17-29.

12. Farrell GC, George J, Hall PM, McCullough AJ. Fatty liver disease: NASH and related disorders. Oxford: Blackwell; 2005.

13. Loguercio C, De Simone T, Federico A, Terracciano F, Tuccillo C, Di Chicco M, et al. Gut-liver axis: a new point of attack to treat chronic liver damage? Am J Gastroenterol. 2002;97:2144-6.

14. Lakhani SV, Shah HN, Alexander K, Finelli FC, Kirkpatrick JR, Koch TR. Small intestinal bacterial overgrowth and thiamine deficiency after Roux-en-Y gastric bypass surgery in obese patients. Nutr Res. 2008;28:293-8.

15. Madrid AM, Poniachik J, Quera R, Defilippi C. Small intestinal clustered contractions and bacterial overgrowth: a frequent finding in obese patients. Dig Dis Sci. 2011;56:155-60.

16. Jung UJ, Choi MS. Obesity and its metabolic complications: the role of adipokines and the relationship between obesity, inflammation, insulin resistance, dyslipidemia and nonalcoholic fatty liver disease. Int J Mol Sci. 2014;15:6184-223.

17. Singh A, Le P, Peerzada MM, Lopez R, Alkhouri N. The utility of noninvasive scores in assessing the prevalence of nonalcoholic fatty liver disease and advanced fibrosis in type 2 diabetic patients. J Clin Gastroenterol. 2017.

18. Bedossa P, Patel K. Biopsy and noninvasive methods to assess progression of nonalcoholic fatty liver disease. Gastroenterology. 2016;150:1811-22.e1814.

19. European Association for Study of Liver; Asociacion Latinoamericana para el Estudio del Higado. EASL-ALEH Clinical Practice Guidelines: Non-invasive tests for evaluation of liver disease severity and prognosis. J Hepatol 2015;63:237-64.

20. Ratziu V, Zelber-Sagi S. Pharmacologic therapy of non-alcoholic steatohepatitis. Clin Liver Dis. 2009;13:667-88.

21. Sanyal AJ, Chalasani N, Kowdley KV, McCullough A, Diehl AM, Bass NM, et al. Pioglitazone, vitamin E, or placebo for nonalcoholic steatohepatitis. N Engl J Med. 2010;362:1675-85.

22. Neuschwander-Tetri BA, Loomba R, Sanyal AJ, Lavine JE, Van Natta ML, Abdelmalek MF, et al. Farnesoid X nuclear receptor ligand obeticholic acid for non-cirrhotic, non-alcoholic steatohepatitis (FLINT): a multicentre, randomised, placebo-controlled trial. Lancet. 2015;385:956-65.

23. Armstrong MJ, Gaunt P, Aithal GP, Barton D, Hull D, Parker R, et al. Liraglutide safety and efficacy in patients with non-alcoholic steatohepatitis (LEAN): a multicentre, double-blind, randomised, placebo-controlled phase 2 study. Lancet. 2016;387:679-90. 